\title{
Forum
}

\section{No science, no success and still no need for captive-origin lion reintroduction: a reply to Abell \& Youldon}

\author{
Luke T.B. Hunter, Paula White, Philipp Henschel, Laurence Frank \\ Cole Burton, Andrew Loveridge, Guy Balme, Christine Breitenmoser and \\ URS BREITENMOSER
}

\begin{abstract}
A bell \& Youldon (2013) claim that restoration of lions using captive-origin animals can contribute to in situ lion conservation, suggesting it is comparable to established methods using wild-caught founders. Their argument hinges on an attempt to discredit using wild lions to restore populations but they ignore the empirical record of longstanding success from this approach. Concomitantly, they produce no data or even a credible justification to support their subjective, impractical faith in captive animals as founders.

Contrary to Abell \& Youldon's implication we do not claim that lion restoration programmes using captiveorigin lions are or will be failures'. We have little doubt that, if enough captive-origin lions were released, some may survive. However, Abell \& Youldon do not provide a meaningful rationale to consider this a legitimate alternative. It is spurious to claim that both captive-origin and wild-born approaches can 'play a part' when the former has wasted millions of dollars and years of effort, elevated the risk to lions and people, and has not established a single, free-ranging lion. We do not dispute that the approach may eventually do so but given the considerable drawbacks, and the evidence-based advantages of using wild lions, it is illogical and unscientific to pursue it. Our argument, simply put, remains that for every objective criterion by which reintroductions are planned and evaluated, wild lions are better candidates for increasing the likelihood of success.
\end{abstract}

Luke T.B. Hunter (Corresponding author), Philipp Henschel, Laurence Frank and GuY Balme Panthera, 8 West 40th Street, 18th Floor, New York, NY 10018, USA. E-mail lhunter@panthera.org

Paula White Center for Tropical Research, University of California, Los Angeles, USA

COLE BURTON Alberta Biodiversity Monitoring Institute, University of Alberta, Edmonton, Canada

ANDREW Loveridge Wildlife Conservation Research Unit, Zoology Department, Oxford University, UK

Christine Breitenmoser and URS Breitenmoser IUCN/SSC Cat Specialist Group, c/o KORA, Muri, Switzerland
We agree with the statement that 'measures need to be taken to ensure the causes of the original decline or loss do not reoccur'. As we noted, identifying and preparing the release site, including mitigating the causes of decline, is an essential first step for reintroducing any large carnivore. This applies whether the founders are wild-caught or captive-origin. Abell \& Youldon's attempt to discredit wild lion translocations by citing two cases where founders died of anthropogenic causes is a diversion. Do they believe captives would somehow be better equipped to avoid the same threats? In fact, carnivores reintroduced from captivity are more likely than wild founders to die of both anthropogenic and natural causes, or are frequently recaptured to avoid death (Jule et al, 2008). Similarly, we are surprised at their suggestion that nothing can be learned about translocations from the extensive literature and experience covering species other than 'large, social felids' (i.e. lions). This claim indicates a dismissal of science and lessons learned from the field in the attempt to justify an impractical approach.

Abell \& Youldon's lengthy discussion on disease and inbreeding issues shows little understanding of in situ experience. Again, they ignore a wealth of results accumulated from 2 decades of wild-wild translocation practice that has not produced disease transmission, mortality, epidemics or any other evidence of the risks they avow. Similarly, they apparently misunderstand our recommendations for managing disease and inbreeding risk when planning translocations, protocols that have successfully fostered population re-establishment (Slotow \& Hunter 2009). Finally, they offer nothing to demonstrate that using captive founders, especially those of mongrel, opportunistic provenance promoted by private owners such as ALERT, is a preferable alternative (see Greenwood et al., 2012, for further evidence of the risks of exotic disease for captive carnivores).

Abell \& Youldon conclude that 'rigorous assessment and application of a range of effective conservation strategies' will help save the African lion. We agree but regrettably they have produced nothing to show that their approach 
qualifies as effective. Simply bundling it with demonstrably practical solutions such as wild-wild translocations does not lend it credence. As with any approach we would expect to see a credible science-based rationale and peer-reviewed results that address the significant disadvantages we catalogued. Abell \& Youldon's response does not bring us any closer to those criteria. Opportunities for lion reintroductions are limited and make a minor contribution to the species' conservation needs (Hunter et al., 2007). The quasi-conservation rationale of the encounter industry misleads the public and policy makers into believing that reintroduction is a panacea to the extremely complex challenges of conserving wild lions. Although paying tourists may enjoy cuddling lion cubs this approach does nothing to address the real issues driving the lion's decline, and diverts valuable human and financial resources that should be devoted to ecosystem-wide protection where wild lions still persist.

\section{References}

Abell, J. \& Youldon, D. (2013) Attending to the 'biological, technical, financial and sociological factors' of lion conservation: a response to Hunter et al.. Oryx, 47, 25-26.

Greenwood, A.D., Tsangaras, K., Ho, S.Y.W., Szentiks, C.A., Nikolin, V.M., MA, G. et al. (2012) A potentially fatal mix of herpes in zoos. Current Biology, 18, 1727-1731.

Hunter, L.T.B, Pretorius, K., Carlisle, L., Rickelton, M., Walker, C., Slotow, R. \& Skinner, J.D. (2007) Restoring lions Panthera leo to Northern KwaZulu-Natal, South Africa: short-term biological and technical success but equivocal long-term conservation. Oryx, 41, 196-204.

Jule, K.R., Leaver, L.A. \& Lea, S.E.G. (2008) The effects of captive experience on reintroduction survival in carnivores: a review and analysis. Biological Conservation, 141, 355-363.

Slotow, R. \& Hunter, L.T.B. (2009) Reintroduction decisions taken at the incorrect social scale devalue their conservation contribution: the African lion in South Africa. In Reintroduction of Top-Order Predators (eds M. Hayward \& M. Somers), pp. 43-71. Wiley-Blackwell, Oxford, UK. 